\title{
Macropropagación de Paulownia elongata x fortunei a partir de esquejes de raíz en la Región del Biobío, Chile
}

\section{Macropropagation of Paulownia elongata x fortunei from root cuttings in the Biobío Region, Chile}

\author{
Gerson Riffo ${ }^{*}$, Fernando Muñoz ${ }^{2}$, Matilde Uribe ${ }^{1}$, Jorge Cancino², Eduardo Acuña ${ }^{2}$ \& Rafael \\ RUBILAR $^{2}$ \\ ${ }^{1}$ Universidad de Concepción, Facultad de Ciencias Forestales y Centro de Biotecnología, Casilla 160-C, Concepción, \\ Chile. \\ ${ }^{2}$ Universidad de Concepción, Facultad de Ciencias Forestales, Casilla 160-C, Concepción, Chile. \\ *geriffo@udec.cl
}

\begin{abstract}
RESUMEN
El cultivo de Paulownia fue recientemente introducido en Chile. En esta investigación se presentan resultados de la propagación vegetativa del híbrido Paulownia elongata x fortunei, utilizando esquejes de raíz. El objetivo de este trabajo fue determinar la capacidad de los esquejes de raíz del híbrido P. elongata $\mathrm{x}$ fortunei de formar brotes adventicios para obtener nuevas plantas, que conserven el genotipo y las características del híbrido mediante una técnica más sencilla y de menor costo. Para ello se utilizaron esquejes de raíz de plantas de un año de edad, obtenidos desde plantaciones experimentales, con el propósito de estudiar el comportamiento de la propagación según tres variables: diámetro de esqueje (tres niveles), tipo de sustrato (dos niveles) y estímulo exógeno con BAP/AIB (dos niveles). Se generaron doce tratamientos, cada uno con tres repeticiones. El mejor resultado se obtuvo con esquejes de diámetro pequeño $(0,65-0,79 \mathrm{~cm})$, plantados en compost:perlita y no tratados con hormonas, generando plantas de $9,44 \mathrm{~cm}$ en un periodo de 50 días. Se concluye que para generar plántulas de Paulownia, la propagación vegetativa mediante el uso de esquejes de raíz de árboles de un año de edad es una opción viable.
\end{abstract}

Palabras clave: Propagación vegetativa, esquejes de raíz, brotes adventicios, macropropagación, Paulownia.

\begin{abstract}
Growing Paulownia was recently introduced in Chile. Results of vegetative propagation of hybrid Paulownia elongata $\mathrm{x}$ fortunei are presented in this research, using root cuttings, The aim of this study was to determine the capacity of root cuttings of hybrid $P$. elongata $\mathrm{x}$ fortunei to form adventitious shoots, to get new plants which conserve the genotype and characteristics of hybrid using a simpler and low-cost technique. For this, were used root cuttings of plants with one year old, obtained from experimental plantings, with the purpose to study the propagation behavior according to three variables: cutting diameter (three levels), substrate type (two levels), and exogenous stimulus BAP/IBA (two levels). Twelve treatments were generated, each one with three repeats. The best result was obtained with small diameter cuttings $(0.65-0.79 \mathrm{~cm})$, planted in compost:perlite and not treated with hormones, generating plants $9.44 \mathrm{~cm}$ of height in a period of 50 days. It can be concluded that to generate Paulownia plantlest, vegetative propagation by cuttings root of one year old trees is a viable option.
\end{abstract}

KEYwords: Vegetative propagation, root cuttings, adventitious shoots, macropropagation, Paulownia.

\section{INTRODUCCIÓN}

Paulownia spp. es el único género maderable de la familia Scrophulariaceae, el cual está representado por nueve especies con amplia distribución en el sur y norte de China (Zhu et al. 1986). Paulownia representa especies de árboles de hoja caduca de rápido crecimiento y de amplia tolerancia a diferentes condiciones de suelo y clima (Wang \& Shogren 1992), se distribuye desde climas tropicales a lugares de temperaturas frías. Las hojas de Paulownia son ricas en nitrógeno, siendo útiles como forraje (Song 1988) y como fertilizante. Las inflorescencias son de gran tamaño, con propiedades melíferas, presentan gran colorido durante la primavera, lo cual le entrega potencial como especie ornamental (Zhu et al. 1986). Los árboles de Paulownia son apropiados para el manejo de plantaciones maderables de corta rotación y no requiere ser replantado ya que se regenera desde brotes epicórmicos (Bergmann 1998), su madera puede ser utilizada para revestimientos de paredes $\mathrm{y}$ techos, marcos fotográficos, persianas de madera, puertas, marcos de ventanas (Beel et al. 2005) y en la industria del papel (Jiménez et al. 2005). Además el género posee 
una alta capacidad de crecimiento en ciclos cortos de producción, generando una alta cantidad de biomasa para energía (Martínez et al. 2010, López et al. 2012). Diferentes especies de Paulownia se han propagado a través de semillas (Turner et al. 1988), esquejes de tallos (Bergmann 1998), esquejes de raíz (Stringer 1994, Ede et al. 1997) y cultivo in vitro (Bergmann \& Moon 1997, Ipekci et al. 2001, Ipekci \& Gozukirmizi 2004, Ozaslan et al. 2005, Corredoira et al. 2008, Castillo-Martínez et al. 2012).

Sin embargo, el desarrollo de plantas de Paulownia a partir de semillas es lento y pueden presentar dormancia (CastilloMartínez et al. 2012), en comparación con otros métodos de propagación, debido a esto se prefiere la propagación vegetativa y el cultivo in vitro, siendo esta última una técnica que requiere equipos, materiales y personal especializado de mayor costo a diferencia de la propagación a través de esquejes. Variadas especies de plantas poseen células en las raíces que son capaces de diferenciarse en nuevos puntos de crecimiento, los que pueden formar nuevos brotes o raíces (Hartmann et al. 2010), esto permite la multiplicación de algunas especies mediante el uso de esquejes de raíz para su cultivo en masa y producir plantas genéticamente uniformes.

En los últimos años se han desarrollado híbridos de Paulownia para mejorar y aumentar la producción de biomasa, estos individuos se han propagado mediante diferentes técnicas de multiplicación in vitro, pero el costo de la producción de plántulas es alto. El objetivo de este trabajo fue determinar la capacidad de los esquejes de raíz del híbrido P. elongata x fortunei de formar brotes adventicios para obtener nuevas plantas, que conserven el genotipo y las características del híbrido mediante una técnica más sencilla y de menor costo.

\section{MATERIALES Y MÉTODOS}

Material Vegetal

El material utilizado para el ensayo fue recolectado en el fundo La Isla (3644'17'’S y 72²6'52”O), comuna de Quillón, Región del Biobío, desde plantaciones experimentales de P. elongata $\mathrm{x}$ fortunei de un año de edad durante el mes de diciembre del año 2012. La obtención de los esquejes se realizó desde el sistema radicular de plantas seleccionadas aleatoriamente, a una distancia mayor a $5 \mathrm{~cm}$ del cuello radicular. El corte de los esquejes se realizó verticalmente con una pala cuadrada de borde afilado. El material obtenido se trasladó a laboratorio envuelto en papel húmedo dentro de cajas plásticas.

\section{DiÁmeTros DE ESQUEJES}

En el laboratorio, un día después de la poda, las raíces se lavaron sólo con agua para eliminar la tierra y se cortaron en segmentos de $5 \mathrm{~cm}$ de longitud, los esquejes obtenidos fueron agrupados en tres clases de diámetros (medido con pie de metro), de acuerdo al material disponible y a la metodología propuesta por Stringer
(1994): pequeños $(0,65-0,79 \mathrm{~cm})$, medianos $(0,80-0,99 \mathrm{~cm})$ y grandes (1,0-1,50 cm) (Fig. 1A).

\section{ESTABLECIMIENTO DE ESQUEJES}

Los esquejes fueron plantados en dos cajas metálicas de igual dimensión (Fig. 1B), una con compost y perlita (relación 3:1) como sustrato y la otra caja, con arena como sustrato. El compost utilizado es una mezcla de corteza de pino insigne y paja de trigo (90 y $10 \%$, respectivamente), con tamaño máximo de partícula de $6 \mathrm{~mm}$. Cada caja fue dividida en 18 partes iguales (unidades experimentales de cinco esquejes), que contiene la combinación de los dos niveles de hormona: sin hormona y solución de 6-bencil aminopurina (BAP) y ácido indol-3butírico (AIB), los tres tamaños de esquejes (grande, mediano y pequeño) y tres repeticiones. En total se conformaron 12 tratamientos con 3 repeticiones por tratamiento ( 2 sustratos $\mathrm{x} 2$ niveles de hormona x 3 tamaños de esquejes x 3 repeticiones). La aplicación de reguladores de crecimiento vegetal consistió en una inmersión rápida de 5 seg en una solución de BAP 1000 ppm y AIB 500 ppm. Los esquejes fueron plantados a $5 \mathrm{~cm}$ de profundidad con separación entre esquejes de $3 \mathrm{~cm}$ (Fig. 1C), se regaron dos veces al día, con excepción de los días de lluvia. El desarrollo del experimento se realizó en el vivero de la Facultad de Ciencias Forestales de la Universidad de Concepción, las cajas fueron protegidas con una malla Raschel negra de $50 \%$ de cobertura.

\section{VARIABLES ESTUDIADAS}

Luego de 50 días de plantados, a cada esqueje se le midió la altura del tallo (con regla métrica), desde la superficie del sustrato hasta el ápice de las plantas. En aquellos esquejes que presentaban más de un tallo solo se midió el de mayor longitud. Se analizó la longitud ( $\mathrm{cm}$ ) del tallo y supervivencia de los esquejes $(\%)$, según clase de diámetro $(\mathrm{cm})$ de esqueje, tipo de sustrato y la acción de reguladores de crecimiento BAP y AIB. Se consideró estaca muerta aquella que presentó pudrición.

\section{ANÁLISIS DE DATOS}

Se aplicó a las variables altura de tallo y supervivencia la prueba ANOVA con tres factores para determinar el efecto de los factores principales (sustrato, tamaño esqueje y hormona), así como sus interacciones. Este análisis se complementa con la aplicación de test de diferencia de medias (Tukey, $P<0,05$ ).

\section{RESULTADOS}

El mayor porcentaje de supervivencia se logró con los esquejes de diámetro grande $(79,6 \%)$, aunque el factor diámetro no tuvo efecto significativo en la supervivencia. Por el contrario, la altura de tallo fue mayor en los esquejes de diámetro pequeño $(4,39$ $\mathrm{cm}$ ) y de manera similar a la supervivencia, no se presentaron efectos significativos entre diámetros (Tabla I). El factor sustrato presentó diferencias significativas en la altura del tallo, no así en 
la supervivencia. Por otra parte, en los tratamientos sin hormona se obtuvo mayor altura y mayor supervivencia (diferenciales de $2,32 \mathrm{~cm}$ y $25,5 \%$, respectivamente), con diferencias significativas respecto a los tratamientos con hormona. Esto indica que la aplicación de hormona influyó negativamente en la altura de tallo y supervivencia (Tabla I). La interacción de los factores solo fue significativa para la variable altura de tallo, tanto para la interacción diámetro x hormona, diámetro x sustrato y sustrato $\mathrm{x}$ hormona (Tabla I).

El tratamiento que alcanzó la mayor altura de tallo fue T2 (esqueje de tamaño pequeño, compost:perlita y $\sin$ hormonas) con 9,44 cm (Fig. 1D) y también representó al de mayor supervivencia (93,3 \%) (Tabla II). El tratamiento con menor altura de tallo y supervivencia de esqueje fue T3 (esqueje de tamaño pequeño, arena y con hormonas) con 0,97 cm y 46,6 \%, respectivamente. La supervivencia en los tratamientos T2, T6 y T10 demostró diferencias significativas con los tratamientos T3 y T5 (Tabla II). La altura de tallo de los tratamientos T2 $(9,44 \mathrm{~cm})$ y $\mathrm{T} 6(8,59 \mathrm{~cm})$ fue significativamente diferente al tratamiento T3 $(0,97 \mathrm{~cm})$ (Tabla II).

TABLA I. Significancia de los principales factores y su interacción según la altura de tallos adventicios y la supervivencia de esquejes de Paulownia elongata $\mathrm{x}$ fortunei.

TABLE I. Significance of principal factors and their interaction by height of adventitious shoots and survival of cuttings of Paulownia elongata $\mathrm{x}$ fortunei.

\begin{tabular}{|c|c|c|c|}
\hline \multicolumn{2}{|c|}{ FACTORES } & ALTURA DE TALLO $(\mathrm{Cm})$ & SUPERVIVENCIA ( $\%)$ \\
\hline & Pequeño & $4,39^{\mathrm{a}}$ & $73,3^{\mathrm{a}}$ \\
\hline Diámetro & Mediano & $3,95^{\text {a }}$ & $71,9^{\mathrm{a}}$ \\
\hline & Grande & $3,59^{\text {a }}$ & $79,6^{\mathrm{a}}$ \\
\hline Sustrato & Compost:perlita & $5,75^{a}$ & $76,6^{\mathrm{a}}$ \\
\hline & Arena & $2,20^{\mathrm{b}}$ & $73,2^{\text {a }}$ \\
\hline Hormona & Sin BAP/AIB & $5,14^{\text {a }}$ & $87,7^{\text {a }}$ \\
\hline Hommonia & Con BAP/AIB & $2,82^{b}$ & $62,2^{b}$ \\
\hline Diám & & $0,002 *$ & $0,3688^{\mathrm{ns}}$ \\
\hline Diám & & $<0,0001 * * *$ & $0,1802^{\mathrm{ns}}$ \\
\hline Sustra & & $<0,0001 * * *$ & $0,0659^{\mathrm{ns}}$ \\
\hline Diám & ona $(P)$ & $0,1583^{\mathrm{ns}}$ & $0,0592^{\mathrm{ns}}$ \\
\hline
\end{tabular}

$P$ : probabilidades límites en ANOVA con tres factores. *: $P<0,05$; **: $P<0,01$; ***: $P<0,001$; ns: $P>0,05$. Letras diferentes en cada columna indican diferencias significativas (Tukey, $P<0,05$ ). / $P$ : probability limits ANOVA with three factors. *: $P<0.05 ; * *: P<0.01 ; * *$ : $P<0.001$; ns: $P>0.05$. Different letters in each column indicate significant differences (Tukey, $P<0.05$ ).

TABLA II. Altura de tallos adventicios y supervivencia obtenida en cada tratamiento con esquejes de raíz de P. elongata x fortunei, después de 50 días de plantados.

TABLE II. Height of adventitious shoots and survival obtained in each treatment with root cuttings of $P$. elongata $\mathrm{x}$ fortunei, 50 days after planting.

\begin{tabular}{|c|c|c|c|c|c|}
\hline Tratamiento & DIÁMETRO ESQUEJE & SUSTRATO & HORMONA & ALTURA DE TALLO $(\mathrm{cm})$ & SUPERVIVENCIA ( $\%)$ \\
\hline $\mathrm{T} 1$ & pequeño & compost:perlita & $\mathrm{C} / \mathrm{H}$ & $4,60^{\mathrm{cb}}$ & $66,6^{a b c}$ \\
\hline $\mathrm{T} 2$ & pequeño & compost:perlita & $\mathrm{S} / \mathrm{H}$ & $9,44^{\text {a }}$ & $93,3^{\text {a }}$ \\
\hline T3 & pequeño & arena & $\mathrm{C} / \mathrm{H}$ & $0,97^{\mathrm{e}}$ & $46,6^{c}$ \\
\hline $\mathrm{T} 4$ & pequeño & arena & $\mathrm{S} / \mathrm{H}$ & 2,53 cde & $86,6^{a b}$ \\
\hline T5 & mediano & compost:perlita & $\mathrm{C} / \mathrm{H}$ & 3,07 bcde & $53,3^{\mathrm{bc}}$ \\
\hline T6 & mediano & compost:perlita & $\mathrm{S} / \mathrm{H}$ & $8,59^{\text {a }}$ & $93,3^{\text {a }}$ \\
\hline $\mathrm{T} 7$ & mediano & arena & $\mathrm{C} / \mathrm{H}$ & $1,84 \mathrm{de}$ & $66,6^{a b c}$ \\
\hline $\mathrm{T} 8$ & mediano & arena & $\mathrm{S} / \mathrm{H}$ & 2,38 cde & $74,4 \mathrm{abc}$ \\
\hline T9 & grande & compost:perlita & $\mathrm{C} / \mathrm{H}$ & 3,49 bcd & $60,0 \mathrm{abc}$ \\
\hline $\mathrm{T} 10$ & grande & compost:perlita & $\mathrm{S} / \mathrm{H}$ & $5,39^{\mathrm{b}}$ & $93,3^{\text {a }}$ \\
\hline $\mathrm{T} 11$ & grande & arena & $\mathrm{C} / \mathrm{H}$ & $2,93^{\text {cde }}$ & 80,0 abc \\
\hline T12 & grande & arena & $\mathrm{S} / \mathrm{H}$ & $2,56^{\text {cde }}$ & $85,0^{a b}$ \\
\hline
\end{tabular}

$\mathrm{BAP}=6$-bencil aminopurina; $\mathrm{AIB}=$ ácido indol-3-butírico. $\mathrm{C} / \mathrm{H}=$ con aplicación exógena de BAP $1000 \mathrm{ppm} / \mathrm{AIB} 500 \mathrm{ppm} ; \mathrm{S} / \mathrm{H}=$ sin la aplicación exógena de BAP $1000 \mathrm{ppm} / \mathrm{AIB} 500 \mathrm{ppm}$. Letras distintas indican diferencias significativas $(P<0,05)$. / BAP $=6$-benzyl aminopurine; IBA = indole-3-butyric acid. $\mathrm{C} / \mathrm{H}=$ with exogenous application BAP $1000 \mathrm{ppm} / \mathrm{IBA} 500 \mathrm{ppm}$; $\mathrm{S} / \mathrm{H}=$ without exogenous application of BAP $1000 \mathrm{ppm} / \mathrm{IBA} 500 \mathrm{ppm}$. Different letters indicate significant differences $(P<0.05)$. 


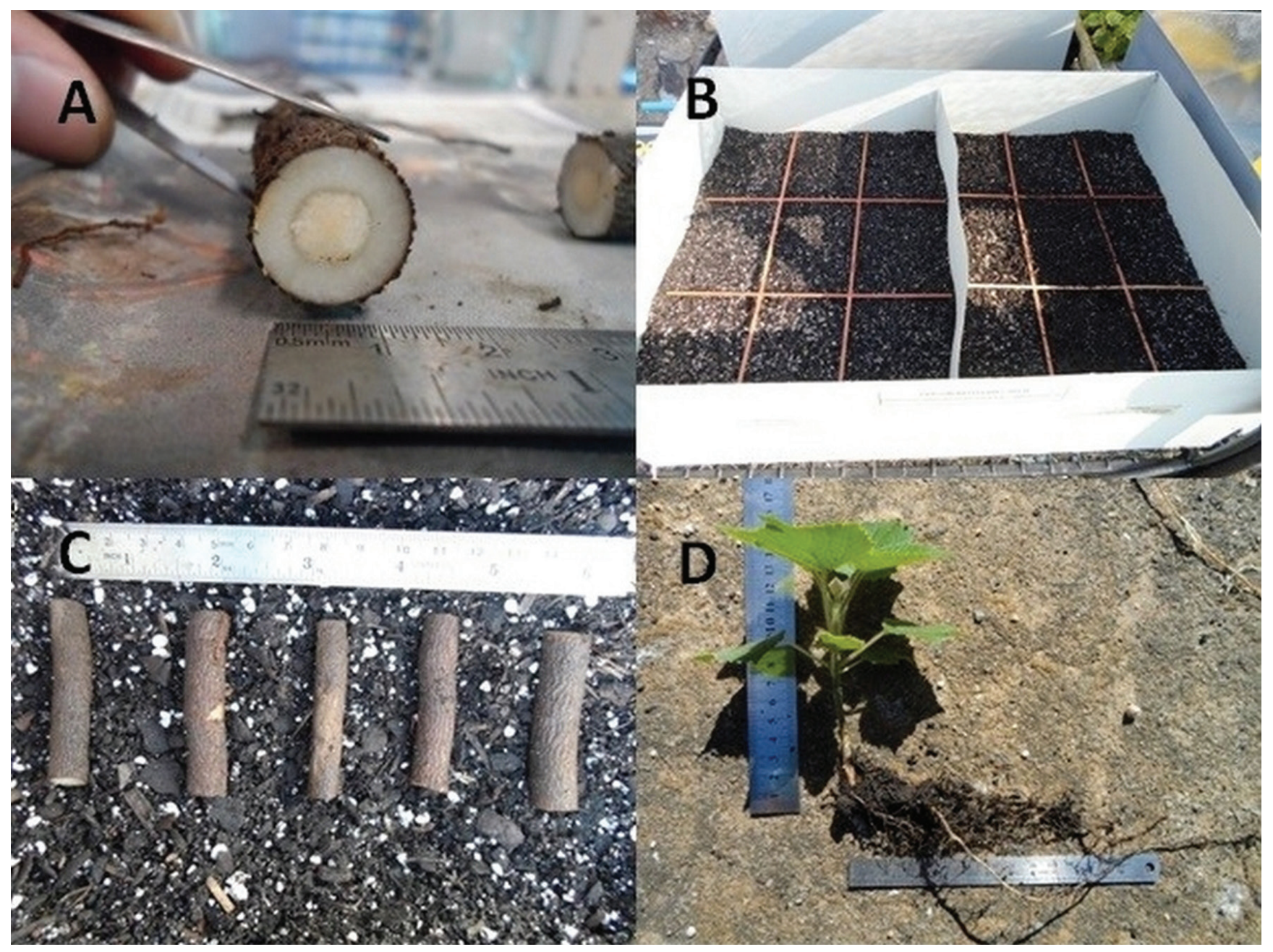

FiguRa 1. Macropropagación de esquejes de raíz de $5 \mathrm{~cm}$ de Paulownia elongata x fortunei. A) corte transversal de esqueje de raíz, B) caja metálica con sustrato compost:perlita, C) posición de los esquejes de raíz en cada unidad experimental, D) brote adventicio desarrollado en el esqueje de raíz después de 50 días de plantado.

FIGURE 1. Macropropagation root cuttings $5 \mathrm{~cm}$ of Paulownia elongata $\mathrm{x}$ fortunei. A) cross section of root cutting, B) metal box with compost: perlite substrate, C) position of the root cuttings in each experimental unit, D) shoot developed adventitious from roots cuttings, 50 days after planting.

\section{DISCUSIÓN}

Los resultados del estudio demuestran que es posible obtener plantas completas de $P$. elongata $\mathrm{x}$ fortunei a partir de esquejes de raíz de $5 \mathrm{~cm}$ de longitud, con diámetro entre 0,65 y $1,5 \mathrm{~cm}$ desde individuos de un año de edad. Lo cual está determinado por las cualidades que posee la especie para generar nuevos órganos adventicios, y las condiciones del material vegetal de origen, edad cronológica, edad fisiológica y la fenología en el momento de la colecta. Se determinó que el diámetro del esqueje no tiene efecto significativo en la altura del tallo, esto significa que incluso los esquejes más delgados tenían el potencial adecuado para regenerar brotes adventicios. El contenido de sustancias de reserva de los esquejes de raíz no fue un factor limitante para generar brotes, sin embargo su ubicación en diferentes sustratos produjo alturas de tallos significativamente diferentes. Similar resultado obtuvo Stringer (1994) con esquejes de $P$. tomentosa (Thunb.) Steud. de diámetro entre 1,3 y $5,1 \mathrm{~cm}$ y longitud entre 7,6 a 22,8 cm. No obstante Ede et al. (1997) obtuvieron resultados que se contradicen con los obtenidos en el presente estudio y el de Stringer (1994), estos autores determinaron que esquejes de raíz de $P$. fortunei y $P$. tomentosa de un año de edad presentaron una fuerte relación entre el diámetro del esqueje y la regeneración de brotes; las mayores alturas de tallos las obtuvieron con esquejes de 5 a $10 \mathrm{~cm}$ de diámetro y longitud de 8 a $10 \mathrm{~cm}$, estos estudios demuestran que para diferentes especies del género Paulownia la propagación vegetativa mediante el uso de esquejes de raíz es una opción viable, si se tiene en cuenta el tamaño del esqueje (diámetro y longitud), para una mejor respuesta a la formación de nuevos órganos adventicios. 
Junto con lo anterior se debe tener presente la fenología de la planta donadora, del material vegetal al momento de colectar los esquejes de raíz (estacionalidad). Uribe et al. (2011) determinaron mayor porcentaje de esquejes de tallo enraizados de Berberidopsis corallina Hook. f. cuando se recolectaron en la época de otoño $(89 \%)$ e invierno $(93 \%)$ a diferencia de las estacas colectadas en verano (44\%).

En otras especies hay resultados contradictorios, en este sentido Ghani \& Cahalan (1991) realizaron un estudio con esquejes de raíz de Prunus avium (L.) L., determinando que diámetros gruesos $(0,7 \mathrm{a} 1,2 \mathrm{~cm})$ presentaron significativamente un mayor número de brotes que esquejes de diámetro delgado $(0,2$ a $0,3 \mathrm{~cm})$. Sin embargo, Stenvall et al. (2006) trabajando con esquejes de raíz del híbrido de álamo (Populus tremula L. $\mathrm{x} P$. tremuloides Michx.) de dos años de edad, determinaron que no hubo relación significativa entre el número de brotes y el diámetro de los esquejes utilizados (obtuvieron mayor cantidad de brotes en los esquejes de diámetro mediano entre 0,31-0,60 cm). El desarrollo de brotes adventicios en raíces se ha observado principalmente en árboles de rápido crecimiento, caso del álamo, donde la formación de brotes adventicios está dada por la disminución en la concentración de auxinas endógenas dentro de la raíz, principalmente por situaciones de estrés como fuego o el corte en los esquejes (Eliasson 1969, Hartmann et al. 2010). Además, la capacidad de regenerar brotes a partir de esquejes de raíz está dada por la cantidad de carbohidratos de reserva presentes en el esqueje al momento de la cosecha de las raíces (Schier \& Zasada 1973, Ghani \& Cahalan 1991, Kays \& Canham 1991, Ede et al. 1997). Junto con esto, la capacidad de regeneración de los esquejes de raíz también está influenciada por la localización del esqueje en el sistema radicular de la planta madre, hecho conocido como topófisis (Hartmann et al. 2010).

Respecto a la polaridad de los esquejes, Ede et al. (1997) observaron que los esquejes de raíz poseen fuerte polaridad tanto en esquejes plantados de forma vertical, inclinados $45^{\circ}$ u horizontalmente, lo cual concuerda con lo observado en el presente ensayo donde los esquejes plantados horizontalmente desarrollaron los nuevos órganos (raíz y tallo) en el extremo de cada esqueje de acuerdo a su polaridad, independientemente de la orientación durante la siembra. Similar situación observaron Stenvall et al. (2006), quienes plantaron los esquejes de raíz de álamo de forma vertical de acuerdo a su polaridad, obteniendo nuevas raíces en el extremo distal y los brotes se forman en el extremo proximal. Esta polaridad resulta del transporte polar de auxinas, basipetalamente de brotes a raíces junto con la distribución asimétrica de la auxina dentro de cada célula (Taiz \& Zeiger 2010).

El presente estudio demostró la capacidad del híbrido P. elongata $\mathrm{x}$ fortunei para formar brotes adventicios desde esquejes de raíz después de 50 días de establecido en sustrato. En estudios posteriores con P. elongata x fortunei se debería investigar el ciclo de poda de raíces para obtener esquejes, el comportamiento en campo de las plantas provenientes de esquejes de raíz y determinar el efecto de sucesivas multiplicaciones vegetativas en el desarrollo de los clones.

\section{AGRADECIMIENTOS}

Los autores agradecen el financiamiento del Proyecto FONDEF B09i 1008 y el apoyo del Centro de Biotecnología de la Universidad de Concepción por las facilidades otorgadas para el desarrollo del ensayo.

\section{BIBLIOGRAFÍA}

Beel, M., S. Davis, J. Murphy \& P. Pirer. 2005. Product potential of Paulownia timber. Australian Forestry 68(1): 3-8.

Bergmann, B. 1998. Propagation method influences first year field survival and growth of Paulownia. New Forests 16: 251264.

Bergmann, B. \& H.K. Moon. 1997. In vitro adventitious shoot production in Paulownia. Plant Cell Reports 16: 315-319.

Castillo-Martínez, C., M.A. Gutiérrez, M. Buenrostro, V. Ceetina \& J. Cadena. 2012. Regeneración de plantas de Paulownia elongata Steud. por organogénesis directa. Revista Mexicana de Ciencias Forestales 2(10): 41-49.

Corredoira, E., A. Ballester \& A.M. Vieitez. 2008. Thidiazuroninduced high-frequency plant regeneration from leaf explants of Paulownia tomentosa mature trees. Plant Cell Tissue and Organ Culture 95: 197-208.

Ede, F.J., M. Auger \& T.G.A. Green. 1997. Optimizing root cutting success in Paulownia spp. Journal of Horticultural Science 72(2): 179-185.

Eliasson, L. 1969. Growth regulators in Populus tremula I. distribution of auxin and growth inhibitors. Physiologia Plantarum 22: 1288-1301.

Ghani, A.K.M.O. \& C.M. Cahalan. 1991. Propagation of Prunus avium from root cuttings. Forestry 64(4): 403-409.

Hartmann, H.T., D.E. Kester, F.T. Davies \& R. Geneve. 2010. Plant Propagation: Principles and Practices, $8^{\text {th }}$ ed. Prentice-Hall, London. 880 pp.

Ipekci, Z., A. Altinkut, K. Kazan, K. Bajrovic \& N. Gozukirmizi. 2001. High frequency plant regeneration from nodal explants of Paulownia elongata. Plant Biology 3: 113-115.

IPEKCI, Z. \& N. GozUKIRMIZI. 2004. Indirect somatic embryogenesis and plant regeneration from leaf and internode explants of Paulownia elongata. Plant Cell Tissue and Organ Culture 79: 341-345.

Jiménez, L., A. Rodríguez, J.L. Ferrer, A. Pérez \& V. Angulo. 2005. La Paulownia: una planta de rápido crecimiento como materia prima para la fabricación de papel. Afinidad 62(516): 100-105.

KAYS, J.S. \& C.D. CANHAM. 1991. Effects of time and frequency of cutting on hardwood root reserves and sprout growth. Forest Science 37(2): 524-539.

López, F., A. Pérez, M.A.M. Zamudio, H.E. De Alva \& J.C. García. 2012. Paulownia as raw material for solid biofuel and cellulose pulp. Biomass and Bioenergy 45: 77-86.

Martínez, E., M.E. Lucas-Borja, M. Andrés-Abellán, F.R. López, A. García \& A. Del Cerro. 2010. Aprovechamiento 
energético de Paulownia spp. en el ámbito mediterráneo. Revista Montes 102: 5-12.

Ozaslan, M., C. CAn \& T. Aytekin. 2005. Effect of explant source on in vitro propagation of Paulownia tomentosa Steud. Biotechnology and Biotechnological Equipment 19(3): 2026.

Schier, G.A. \& J.C. ZASADA. 1973. Role of carbohydrate reserves in the development of root suckers in Populus tremuloides. Canadian Journal of Forest Research 3(2): 243-250.

Song, Y. 1988. Nutritive components of Paulownia leaves as fodder. Chemical Industry and Forest Products 8(3): 44-49.

Stenvall, N., T. Haapala, P. Pulkkinen. 2006. The role of a root cutting's diameter and location on the regeneration ability of hybrid aspen. Forest Ecology and Management 237: 150-155.

Stringer, J. 1994. Sprouting and growth of Paulownia tomentosa root cuttings. Tree Planters' Notes 45(3): 95-100.
Taiz, L. \& E. Zeiger. 2010. Plant Physiology, $5^{\text {th }}$ ed. Sinauer Associates, USA.782 pp.

Turner, G.D., R. LAU \& D. Young. 1988. Effect of acidity on germination and seedling growth of Paulownia tomentosa. Journal of Applied Ecology 25: 561-567.

Uribe, M., R. Durán, G. Bravo, F. Mora, P. Cartes \& C. Delaveau. 2011. Propagación vegetativa de Berberidopsis corallina Hook.f., una especie en peligro de extinción, endémica de Chile. Gayana Botánica 68(2): 135-140.

Wang, Q. \& J.F. Shogren. 1992. Characteristics of the cropPaulownia system in China. Agriculture, Ecosystems \& Environment 39(3-4): 145-152.

Zhu, Z.H., C.J. Chaо, X.Y. Lu \& Y.G. Xiong. 1986. Paulownia in China: cultivation and utilization. Asian Network for Biological Sciences and International Development Research Center, Singapure. 65 pp.

Recibido: 26.05 .14

Aceptado: 07.01.15 